\title{
Resectability and Vascular Management of Retroperitoneal Gynecological Malignancies: A Large Single-institution Case-Series
}

\author{
GIOVANNI TINELLI ${ }^{1}$, SERENA CAPPUCCIO $^{2}$, EZIO PARENTE$^{1}$, ANNA FAGOTTI $^{2}$, \\ VALERIO GALLOTTA ${ }^{2}$, CARMINE CONTE ${ }^{2}$, BARBARA COSTANTINI ${ }^{2}$, \\ SALVATORE GUELI ALLETTI ${ }^{2}$, GIOVANNI SCAMBIA ${ }^{2}$ and GIUSEPPE VIZZIELLI ${ }^{2}$ \\ ${ }^{1}$ Division of Vascular Surgery, "Agostino Gemelli" Foundation University Hospital, Catholic University, Rome, Italy; \\ ${ }^{2}$ Department of Women's and Children's Health, "Agostino Gemelli" Foundation University Hospital, \\ Catholic University, Rome, Italy
}

\begin{abstract}
Background/Aim: To report on morbidity and oncological outcomes in a consecutive series of gynecological malignancies involving the vascular district. Patients and Methods: We retrospectively evaluated a consecutive series between 1/2015 and 1/2017 with suspicious gynecological malignancies involving the vascular district. Peri-operative data and survival rates were computed. Results: Eighthundred-four women with gynecological malignancies were admitted for major oncologic surgery during the study period, and among them, 50 cases (6.2\%) showed vascular involvement. Twenty-seven and 23 patients were submitted to minor and major vascular procedures, respectively. RO resection was achieved in 44 patients. There were no perioperative mortalities. Major postoperative complications occurred in 6 patients (12.0\%). The 2-year disease free survival (DFS) was $67 \%$ if $R 0$ resection was achieved. In patients with positive pathological margins $(n=2)$, the 2-year DFS was 33\%. Conclusion: Vascular procedures can be safely performed with a proper pre-operative planning and may not be an impediment to major gynecological oncological surgery.
\end{abstract}

In the last decades, the treatment of gynecological tumors has evolved toward a multimodal approach, including different combinations of chemotherapy, radiotherapy, and surgery, to obtain an improved oncological outcome (1-2).

Correspondence to: Dr. Giuseppe Vizzielli, Division of Gynecologic Oncology, "Agostino Gemelli” Foundation University Hospital, Rome, Italy. Tel: +39 0630154979, Fax: +39 063052132, e-mail: giuseppevizzielli@yahoo.it; giuseppe.vizzielli@policlinicogemelli.it

Key Words: Vascular resection, gynecological malignancies, postoperative complications.
Radical surgery, which is a milestone in the treatment of these malignancies, may require tailoring the extension of the intervention to other non-gynecological structures (3-8). Among them, one of the most important districts connected with major gynecological oncology surgery is the vascular one (9-11). The close anatomical proximity of the female genital tract and their lymphatic drainages with aortoiliac pathways may hesitate into accidental operative injuries to the iliac vessels, aorta and vena cava and/or combinations of these during gynecological surgery. Moreover, the complete eradication of the tumor may require for the partial or total removal of vascular structures with subsequent reconstructive vascular procedures (9-11).

Taking into account these considerations, an appropriate and integrated surgical strategy seems to be crucial for the treatment of gynecological cancers. In this context, vascular procedures appear complementary to the treatment, with both a demolitive and reconstructive intent.

Nevertheless, the role of the vascular surgery in gynecologic oncology has not been completely encoded yet. With the aim to address this specific issue, we evaluated the early and late post-operative complications, as well as the clinical outcome of a retrospective, single-Institution series of women with gynecological malignancies, involved the vascular district.

\section{Patients and Methods}

After obtaining Institutional Review Board approval (study protocol reference number 31883/17, 16 March 2017), clinical charts of women with gynecological cancers consecutively admitted to the Division of Gynecologic Oncology, “Agostino Gemelli” Foundation University Hospital, Rome, Italy, between January 2015 and January 2017, were retrospectively analyzed. Patients' demographics, preoperative, surgical, and postoperative data were collected. Only patients submitted to major gynecological oncologic surgery were 
considered for this study. Major surgical procedures were taken into consideration when pelvic and/or para-aortic lymphadenectomy and/or bowel resection and/or diaphragmatic stripping/ resection and/or omentectomy and/or peritonectomy and/or urological surgery were performed. Tumor size, the extension to pelvic anatomical structures, and absence of distant metastatic disease were assessed by preoperative imaging (CT, MRI, and PET-CT scans).

More in depth, the vascular pre-operative evaluation was performed through a high-resolution CT scan with slice thickness greater than $1.00 \mathrm{~mm}$ and a good injection quality in arterial and venous time series. A 3D-Workstations, allowing multiplanar, curvilinear and $3 \mathrm{D}$ reconstructions of pre- operative images, are routinely used.

The involvement of the large vascular structures (common and/or external iliac vessels, aorta and/or vena cava) was pre-operatively assessed according to our Institutional categorization (i.e. Tinelli's score, named after our consultant vascular surgeon) after CT examination (4). It was assumed the need of vascular reconstruction for preoperative T4 (Figure 1).

Vascular procedures considered for the analysis were divided into minor (i.e., sutures of vascular damage) and major (tangential resections, major vessels' resections, graft selection/sizing and reconstruction with conduit).

Agreeing to internal policy, a priori all women with a performance status $\geq 3$ according to ECOG (i.e. Eastern Cooperative Oncology Group) were excluded from the major vascular surgery and/or other major surgical procedures (12), but could undergo other needed procedures according to surgeons and anesthesiologist's judgments.

The type of residual tumor after resection was defined based on the status of the final pathologic margins: R0, microscopically negative; R1, microscopically positive; and R2, macroscopically (grossly) positive. All cases were performed as a multidisciplinary surgical effort (i.e. a gynecologic oncologist worked with surgeons from the urology, vascular, and plastic services, as well as a radiation oncologist if needed). In no case, the vascular impairment has been considered a priori a judgment of inoperability.

Surgical technique. Tumors were resected with a meticulous dissection with vessels respect when was possible (Figure 2). Alternatively, an en bloc resection together with blood vessels was performed according to the type of vascular involvement and the surgical standards (9). Blood flow was restored concerning the site of resection, the degree of the vascular damage and collateral blood flow (e.g. venous drainage). Arterial reconstructions were usually performed by the appropriate method, such as primary anastomosis, reinsertion, autologous vein (reversed great saphenous vein) was always used when possible or synthetic prostheses (expanded polytetrafluoroethylene [ePTFE] or Dacron), preferably in an anatomic position.

A ligature of vena cava before the renal confluence was performed when more of $2 / 3$ of circumference was affected in the impossibility of a primary anastomosis. For localized defects of the inferior vena cava, we performed a venoplasty (longitudinal suture). Veins obstructed by thrombosis were not reconstructed (ligation of the proximal and distal venous stump). Patients routinely received prophylactic antibiotic treatment (cephalosporin, generally cefazolin) both preoperatively and postoperatively; in case of bowel resection another antibiotic was added (i.e. metronidazole). Bowel preparations were not routinely administered. Silver-containing prostheses or grafts with antibiotic pre-treatment were preferred in case of synchronous large bowel resection in the absence of autologous vein. After intestinal anastomosis, gloves were changed and protecting towels as well as instruments replaced. Perioperatively, a low-dose regimen of heparin was administered (unfractionated heparin intraoperatively and low-molecular-weight heparin in the postoperative phase) and a fulllength compression stocking. Operative time was calculated starting from the skin incision to the end of all surgical procedures. Postoperative complications were obtained from the records of all hospital admissions, discharge summaries, and office visits up to 180 days post-surgery. Complications were graded using the Memorial Sloan Kettering Cancer Center grading system (13). Briefly, Grade 1 (G1) complications required oral medications or bedside interventions, Grade 2 (G2) complications required intravenous medications, enteral or parenteral nutrition, or chest tube insertion, Grade 3 (G3) complications required surgical or radiological intervention, intubation, or therapeutic endoscopy. Grade 4 (G4) complications produced a chronic disability requiring major rehabilitation or organ resection. Grade 5 (G5) complications resulted in death. A major complication was defined as any G3-G5 complication.

An interdisciplinary tumor board was established to evaluate whether additional therapeutic modalities such as radiotherapy or chemotherapy were required.

After discharge, women were regularly seen during the observation period at our outpatient clinic. The standard vascular follow-up was at 30 days and six months postoperatively and yearly after that. Patients were also seen according to the routine oncologic follow-up schedule. They were questioned about symptoms suggesting graft thrombosis, including pain and discomfort. Clinical examinations were usually combined with duplex ultrasonography to evaluate the patency of arterial and venous reconstructions. Besides, CT follow-up examinations for tumor staging were used to assess the patency of arterial and venous reconstructions.

Statistical analysis. Disease-free survival (DFS) was defined as the interval from the diagnosis to recurrence/progression of disease. Overall survival (OS) and DFS were estimated using the KaplanMeier method. Due to the small number of patients and the heterogeneity of the disease in our study, no statistical tests were performed to compare the survival of the different subgroups. All statistical tests were two-sided and differences were considered significant at a level of $p<0.05$. The SPSS statistical software program, version 17.0 (SPSS Inc., Chicago, IL, USA) was used.

\section{Results}

Eight hundred and four women with gynecological malignancies were admitted for major oncologic surgery during the study period and among them, 50 cases (6.2\%) showed vascular involvement at pre-operative imaging. No false positive concerning CT scan were observed and only eleven women have preoperative symptoms that might have aroused suspicion of vascular involvement (data not shown).

Fifteen cases $(30.0 \%)$ showed a preoperative involvement of both artery and vein (i.e. Tinelli's score $\geq 4$ ) and no false positive cases were detected at pre-operative imaging (Table I, Figure 3). No preoperative statistically significant differences were observed between women submitted to minor $(n=27)$ and major vascular surgery $(n=23)$, as shown 


\section{Grading of vascular infiltration}
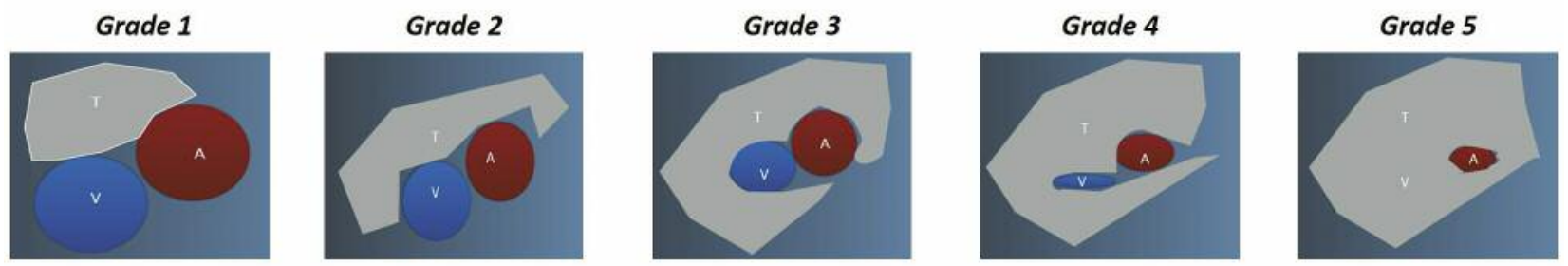

\begin{tabular}{|c|c|}
\hline Grading & Definition \\
\hline 1 & Tumor that border the great vessels without infiltrate the vascular wall \\
\hline 2 & $\begin{array}{l}\text { Tumor that surrounds the great vessels of less than } 50 \% \text { of their circumference, without involvement the } \\
\text { vascular wall }\end{array}$ \\
\hline 3 & $\begin{array}{l}\text { Tumor that surrounds the great vessels of more than } 50 \% \text { of their circumference, with possible } \\
\text { involvement of the vascular wall but with no vascular compression }\end{array}$ \\
\hline 4 & $\begin{array}{l}\text { Tumor that surrounds the great vessels of more than } 50 \% \text { of their circumference, with possible } \\
\text { involvement of the vascular wall and with venous vascular compression }\end{array}$ \\
\hline 5 & tumor completely surrounding the big vessels and totally obliterating them \\
\hline
\end{tabular}

Figure 1. Pre-operative vascular assessment according to Institutional categorization (i.e.: Tinelli's Score) (4).
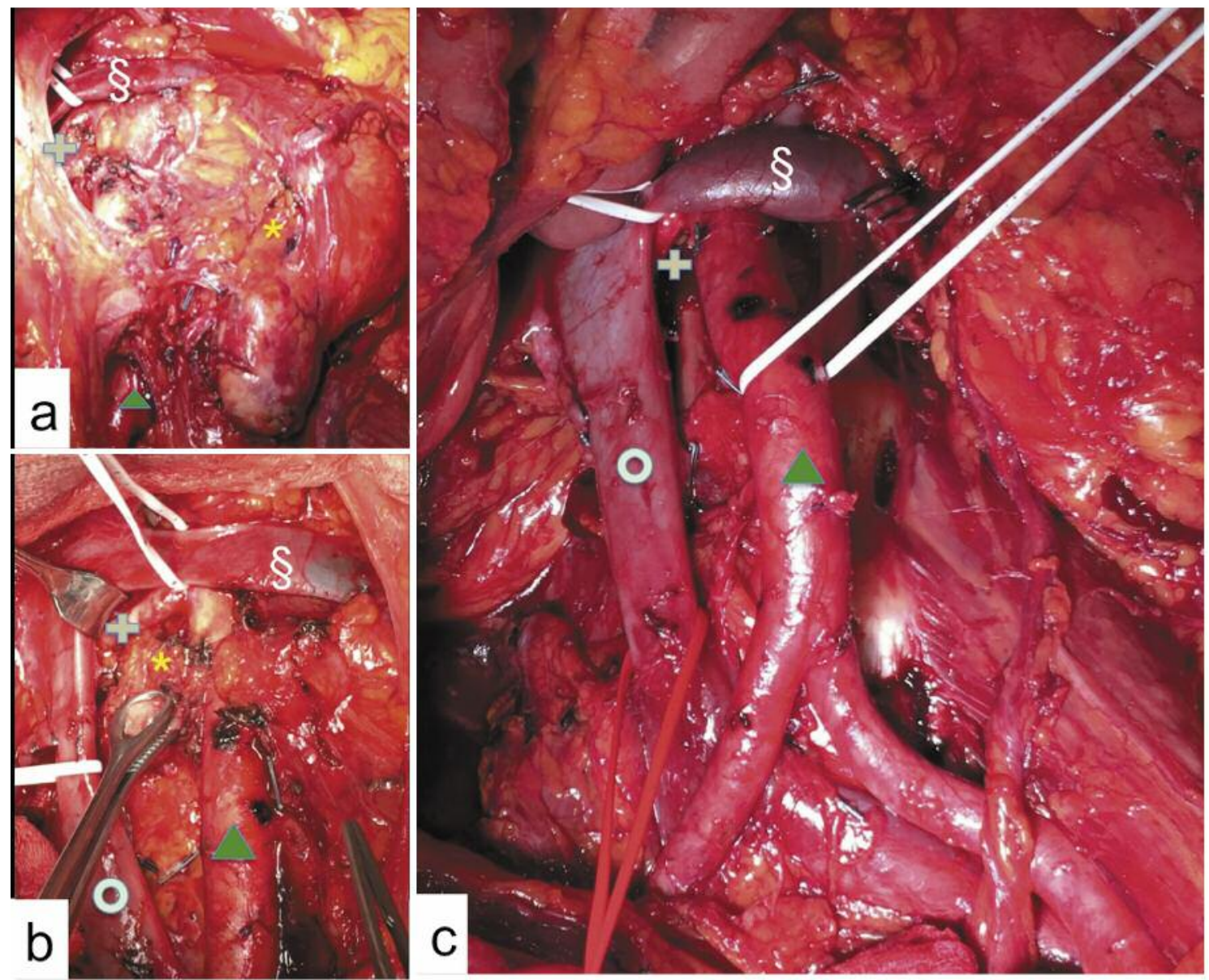

Figure 2. Inter aorto-cava and para-aortic metastatic lymph node: before the tumor dissection (a); detail moment of lymph node between and below the cava and aorta and under the right renal artery (b); final dissection with the free vessels (c). Yellow star: metastatic lymph mass. Green triangle: abdominal aorta; white circle: cava vein; paragraph: left renal vein; gray cross: right renal artery. 


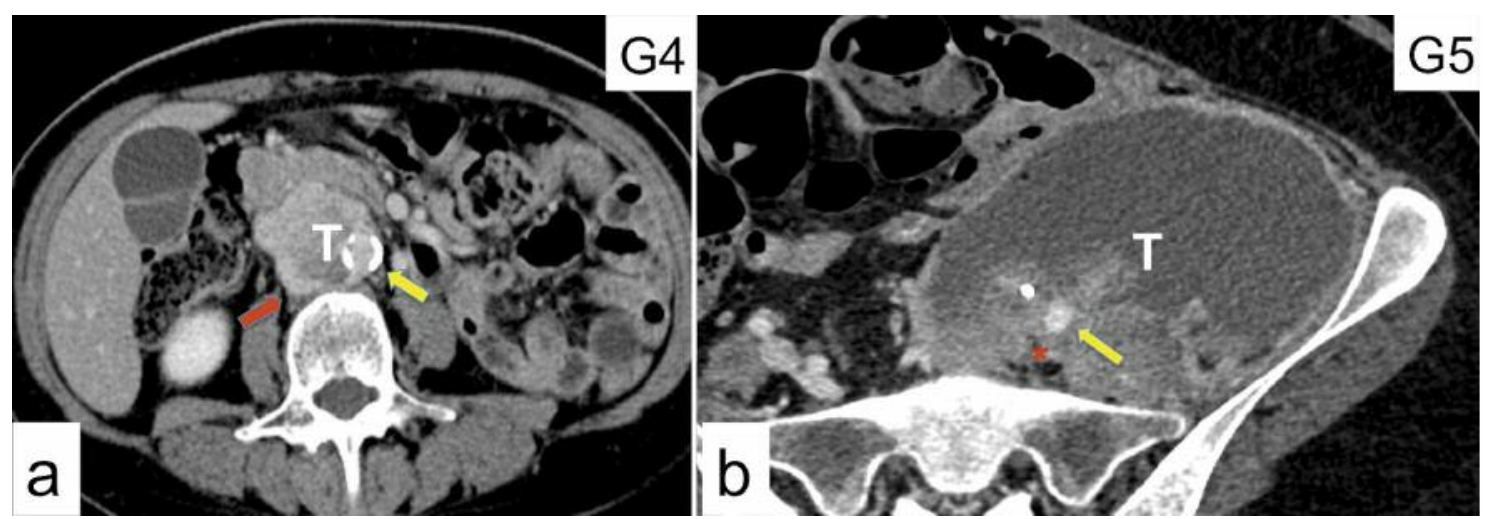

Figure 3. CT axial view of two example of Tinelli's score: Grade 4 with a $>50 \%$ of abdominal aorta involvement (yellow arrow) and vena cava compression (red arrow) (a) and Grade 5, the tumor (T) completely surround the vessels with the left common iliac vein obliteration (red cross) and initial left iliac artery compression (yellow arrow) (b).

A

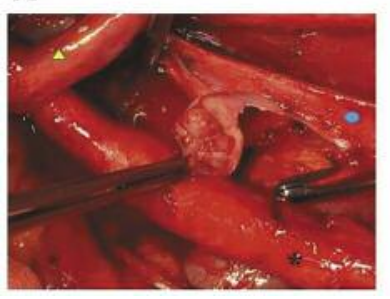

B

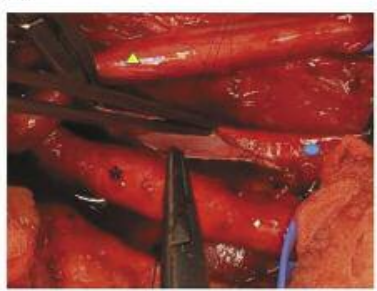

C

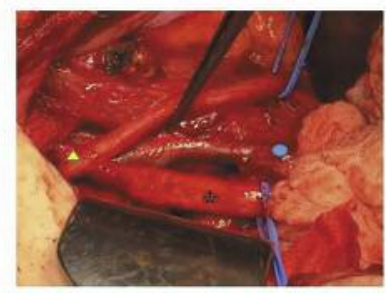

Figure 4. Intraoperative photographs following lymphadenectomy with intravascular tumor dissemination (external iliac vein). A. Tangential resection of external iliac vein. B, C. Reconstruction of the external iliac vein with residual blood vessel diameter $\geq 70 \%$. Yellow triangle: ureter; Blu circle: iliac vein; Black star: iliac artery.

in Table I. The median age was 57 years, with eight patients (16\%) aged $>70$ years at the time of surgery. The overall median BMI was $26 \mathrm{~kg} / \mathrm{m}^{2}$ (range $=17-34 \mathrm{~kg} / \mathrm{m}^{2}$ ). Most patients had ovarian cancer $(61 \%)$, twenty-six women $(51 \%)$ showed a gynecological recurrence, whereas 22 (44\%) of them were submitted to neoadjuvant treatment before the surgery (Table I). Figure 4A illustrates the typical appearance of a blood vessel infiltration. In the same patient, tumor extirpation and its reconstruction are demonstrated by intraoperative photographs in Figure 4B and C.

Perioperative outcomes are shown in Table II. The median overall estimated blood loss was $600 \mathrm{ml}$ (range $=100-2700$ $\mathrm{ml}$ ), with significant differences if a major vascular procedure was performed $(p=0.0001)$. The median operative time, calculated from the beginning of intraperitoneal procedures to skin closure, was $300 \mathrm{~min}$ and $420 \mathrm{~min}$ for minor and major vascular surgery, respectively $(p=0.0001)$. The median time to discharge from the hospital was postoperative days 4 (range $=1-17$ days) and 7 (range $=4-31$ days) for minor and major vascular surgery, respectively $(p=0.003)$.
Vascular major surgery and synchronous major procedure. Twenty-three patients experienced major vascular surgery. Among them, most (56.5\%) were submitted to synchronous resection of pelvic side wall (PSW) musculature with iliopsoas excision $(8.7 \%$ ) and/or urologic structures $(17.4 \%)$ and/or bowel resection (45.5\%).

The vascular surgery details were as follows (Table III): eighteen women had an arterial involvement and among them, seven $(39 \%)$ were treated by arterial resection (Table IV). All arterial resections were followed by vessel reconstruction. In $6(85.7 \%)$ of 7 reconstructions, synthetic grafts were used, and one re-implantation of the renal artery was done. No graft infection was observed.

On the other hand, ten patients (43.4\%) had venous involvement: seven with arterial involvement also and three with only venous involvement. In other two cases, extensive tumor growth precluded venous resection. Venous resection was used in 3 patients (30\%). Resections were reconstructed in 1 of the patients $(10 \%)$ with venous involvement alone. In the other two patients with resection of the external iliac vein, 
Table I. Patients' clinical-pathological characteristics.

\begin{tabular}{|c|c|c|c|c|}
\hline Characteristics & All cases & Minor vascular procedures ${ }^{\mathrm{a}}$ & Major vascular procedures ${ }^{b}$ & $p$-Value \\
\hline All cases & 50 & $27(54.0)$ & $23(46.0)$ & - \\
\hline Median Age (Years)(Range) & $57(21-76)$ & $60(21-76)$ & $57(31-70)$ & 0.061 \\
\hline Median BMI (Kg/mq)(Range) & $26(17-34)$ & $26(18-34)$ & $26(17-34)$ & 0.606 \\
\hline \multicolumn{5}{|l|}{ Primary tumor } \\
\hline Ovarian cancer & $31(61)$ & $17(63.0)$ & $14(60.9)$ & \multirow{4}{*}{0.513} \\
\hline Endometrial cancer & $9(18)$ & $6(22.2)$ & $3(13.0)$ & \\
\hline Cervical cancer & $7(14)$ & $2(7.4)$ & $5(21.7)$ & \\
\hline Vulvar cancer & $3(6)$ & $2(7.4)$ & $1(4.3)$ & \\
\hline \multicolumn{5}{|l|}{ Recurrence } \\
\hline Yes & $26(52)$ & $15(55.6)$ & $9(39.1)$ & \multirow[t]{2}{*}{0.247} \\
\hline No & $24(48)$ & $12(44.4)$ & $14(60.9)$ & \\
\hline \multicolumn{5}{|l|}{ Previous neoadjuvant treatment ${ }^{\mathrm{c}}$} \\
\hline Yes & $22(44)$ & $15(55.6)$ & $13(56.5)$ & \multirow[b]{2}{*}{0.945} \\
\hline No & $28(56)$ & $12(44.4)$ & $10(43.5)$ & \\
\hline \multicolumn{5}{|l|}{ Tinelli's score } \\
\hline$<4$ & $35(70)$ & $22(81.5)$ & $13(56.5)$ & \multirow[t]{2}{*}{0.05} \\
\hline$\geq 4$ & $15(30)$ & $5(18.5)$ & $10(43.5)$ & \\
\hline
\end{tabular}

aSuture of vascular injury; bfrom tangential vascular resection to major vascular reconstruction; ${ }^{\mathrm{c}}$ chemotherapy or radiotherapy.

Table II. Surgical and postoperative characteristics.

\begin{tabular}{|c|c|c|c|c|}
\hline Variables & All cases & Minor vascular procedure ${ }^{a}$ & Major vascular procedures ${ }^{b}$ & $p$-Value \\
\hline All cases & 50 & $27(54.0)$ & $23(46.0)$ & - \\
\hline Median operating time (min) (range) & $355(160-720)$ & $300(160-620)$ & $420(300-720)$ & 0.0001 \\
\hline Median Estimated Blood Loss (mL) (range) & $600(100-2700)$ & $300(100-1000)$ & $1000(300-2700)$ & 0.004 \\
\hline Major structures synchronous resected (\%) & $23(46.0)$ & $10(37.0)$ & $13(56.5)$ & 0.255 \\
\hline Pelvic side wall muscle $(\mathrm{N})$ & $3(6.0)$ & $1(3.7)$ & $2(8.7)$ & 0.459 \\
\hline Urologic structures $^{\mathrm{c}}(\mathrm{N})$ & $7(14.0)$ & $3(11.1)$ & $4(17.4)$ & 0.524 \\
\hline Bowel $(\mathrm{N})$ & $18(36.0)$ & $8(29.6)$ & $10(45.5)$ & 0.309 \\
\hline \multicolumn{5}{|l|}{ Resection Margins (\%) } \\
\hline Microscopically negative (R0) & $44(88.0)$ & $23(85.2)$ & $21(91.3)$ & 0.507 \\
\hline Microscopically positive (R1) & $1(2.0)$ & $1(3.7)$ & 0 & 0.351 \\
\hline Grossly positive (R2) & $5(10.0)$ & $3(11.1)$ & $2(8.7)$ & 0.674 \\
\hline Median Length of hospital stay (days) (range) & $6(1-31)$ & $4(1-17)$ & $7(4-31)$ & 0.003 \\
\hline \multicolumn{5}{|l|}{ Major post-operative complications ${ }^{\mathrm{d}}$} \\
\hline Yes & $6(12.0)$ & $3(11.1)$ & $3(13.0)$ & 0.834 \\
\hline No & $44(88.0)$ & 24 (88.9) & $20(87.0)$ & \\
\hline
\end{tabular}

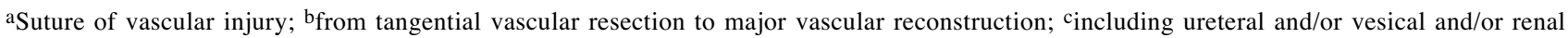

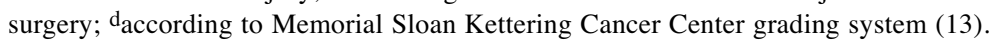

the vessels could not be reconstructed because of massive scarring in the operation field. Of the remaining seven women, venous repair was completed in all cases $(70 \%)$.

Surgical margins and post-operative complications. R0 resection was achieved in 44 patients $(88 \%)$, while the pathologic margins were microscopically ( $\mathrm{R} 1$ resection) and macroscopically (R2 resection) involved in 1 patients $(2.0 \%)$ and $5(10.0 \%)$ patients, respectively. In the $\mathrm{R} 1$ resection case, the surgical margins were too close to the tumor $(<1 \mathrm{~mm})$ at the final pathological examination. Considering women with $\mathrm{R} 2$ resection, in two cases a clear delineation of the tumor borders was suboptimal due to radiation fibrosis; thus, although the final pathologic examination revealed macroscopically (grossly) positive margins, the intraoperative impression was that a complete gross tumor resection was achieved. In the other three cases, an infiltration of the celiac trunk was observed, overcoming the usefulness of a maximal surgical effort with vascular resection. 
Table III. Vascular surgery details.

\begin{tabular}{lc}
\hline Characteristics & No. $(\%)$ \\
\hline All cases & $23(100)$ \\
Vascular procedures & \\
Arterial & $18(78.3)$ \\
Venous & $10(43.5)$ \\
Arterial and Venous & $7(30.4)$ \\
Vessels involved & \\
Inferior Vena Cava & $5(21.7)$ \\
Iliac vein & $5(21.7)$ \\
Mesenteric vein & $1(4.3)$ \\
Aorta & $3(13.0)$ \\
Iliac artery & $10(43.5)$ \\
Mesenteric artery & $5(21.7)$ \\
\hline
\end{tabular}

Table IV. Reconstruction and/or graft material according to the type of vascular involvement.

\begin{tabular}{lcc}
\hline Vascular reconstruction (N=14) & Artery (N) & Vein (N) \\
\hline ePTFE* prosthesis & Iliac (3) & - \\
Dacron Prothesis & Iliac (1) & - \\
ePTFE patch & Aorta (2) & - \\
Venoplasty & - & Vena Cava (1) \\
Venous patch & - & Iliac (5) \\
Reimplantation & Renal artery (1) & - \\
Anastomosis & Iliac (1) & - \\
\hline
\end{tabular}

*ePTFE: Expanded polytetrafluoroethylene.

There were no cases of intra- or postoperative mortality in our series. Thirty-day severe morbidity (grade 3-4) was seen in 6 patients $(12 \%)$ with no statistical differences between major and minor vascular surgery (Table II). The post-operative complications details were as follow (Table V): Wound dehiscence with VAC $^{\circledR}$ application, 1 patients; symptomatic pleural effusion requiring thoracic drainage, 1 patient; pelvic abscess needing abdominal percutaneous drainage, 2 patients; leak of bowel anastomosis with subsequent re-intervention, 1 patient; thrombosis of external iliac artery after 3 hours from the end of operation with subsequent aorto-femoral by-pass, 1 patient. Moreover, no anastomotic leakage associated with blood vessel reconstruction was observed.

Major late complications (31-180 postoperative days) occurred in 2 patients (4\%), and the highest complication grade observed was G3. A summary of the different complications is presented in Table V.

However, the main post-operative late complication was the leg edema (classified as G2 according to MSKCC grading system) that occurred in $10 / 50$ (20\%) women whereas interestingly no post-operative claudication was
Table V. Major postoperative (G3 or more) complications details*.

\begin{tabular}{lcc}
\hline Type of complications & $\begin{array}{c}\text { Early period } \\
(0-30 \text { days })\end{array}$ & $\begin{array}{c}\text { Late Period } \\
(31-180 \text { days })\end{array}$ \\
\hline All cases & 50 & 50 \\
Infectious & $2(4 \%)$ & - \\
$\quad$ Pelvic abscess & $1(2 \%)$ & - \\
$\quad$ Wound infections & $1(2 \%)$ & - \\
Other & $1(2 \%)$ & - \\
Pleural effusion & - & $1(2 \%)$ \\
Leak bowel anastomosis & - & $1(2 \%)$ \\
Decubitus ulcer & - & $1(2 \%)$ \\
Delirium & $1(2 \%)$ & - \\
Neuropathy & - & - \\
Hematoma/Hemorrhage & & \\
Thrombosed vascular graft & & \\
\hline
\end{tabular}

*Major complications affected six women (12\%).

observed (data not shown).

Survival analysis. At the time of data retrieval, 36 patients (72\%) were alive with no evidence of disease, 7 patients (14\%) were alive with disease, and 7 patients (14\%) had deceased from the disease. The median follow-up time for the entire series was 13 months (range=3-27 months). The 2-year DFS was $67 \%$ (95\%CI, not calculable [NC]) for all patients, and the median DFS was 15 months (95\%CI, NC). The 2-year overall survival (OS) was $81 \%$ (95\%CI, NC), and the median OS was not reached $(95 \% \mathrm{CI}, \mathrm{NC})$. However, considering the $\mathrm{R} 0$ resections $(\mathrm{n}=44)$, the 2-year DFS remains about $67 \%(95 \% \mathrm{CI}, \mathrm{NC})$, with a corresponding median OS not reached $(95 \% \mathrm{CI}, \mathrm{NC})$. On the other hand, in patients with R1 plus R2 resections $(n=6)$, the 2-year DFS was only $33 \%$ with a corresponding median OS of 11 months $(95 \% \mathrm{CI}, \mathrm{NC})$.

\section{Discussion}

In recent years, the role of radical surgery aimed to remove pelvic and abdominal disease has been consolidated, both in primary and recurrent gynecological cancers (1-8). Indeed, a certain percentage of gynecological tumor involves major retroperitoneal blood vessels, such as the inferior vena cava, aorta, iliac or visceral vessels, requiring planned vascular resection (9-11).

In this study, we analyzed a subset of patients that underwent major gynecological surgery with synchronous vascular procedures; at our knowledge, there is a lack of studies concerning the frequency and the relevance of vascular surgery in gynecological oncology and this paper represents the first and largest experience in this framework. 
Although the overall vascular procedures could be relatively low in the gynecological setting, the comprehension of this phenomenon may allow a correct approach to such patients requiring a tailored treatment.

In this context, several considerations should be done on the peri-operative factors. First, pre-operative CT-scan (i.e. the Tinelli's score) is the major and easily reproducible factor which allows the suspicion of vessel's involvement with the consequent need of vascular surgeon' skills.

Second, evaluating pre- and intra-operative parameters able to influence the carrying out of major vascular procedures (Table I), highlights that their occurrence is not significantly different in relation to both the primary neoplasm and/or to the time of surgery. Indeed, contrary to what one might have expected, fibrosis and loss of the anatomic cleavage plane due to neoadjuvant treatment and/or recurrent disease do not seem to correlate with major vascular intervention.

Third, the analysis of the major complications represented an additional interesting issue requiring further consideration. In literature, overall complications after major gynecological procedures reached a percentage between $3 \%$ and $50 \%$ (14-16). In our series, no perioperative mortalities were observed and only $6(12 \%)$ patients experienced major postoperative complications, with only 1 (2\%) case of vascular complications (2\%), slightly lower concerning those described in other reports (9). Maybe, an accurate selection of patients together with a multidisciplinary approach has contributed to a more favorable toxicity than expected.

Bleeding was significantly increased in the major vascular procedures; therefore, careful hemostasis and cautious intraoperative administration of heparin are needed. Because patients usually have an excellent peripheral run off, low-dose heparinization is adequate. Moreover, the use of prosthetic vascular grafts after multivisceral resections or bowel surgery was not associated with an increased infectious morbidity, confirming other literature data (9).

We acknowledge that our study is limited by the heterogeneity of the primary tumors and the wide variety of procedures performed. Moreover, the relatively low number of patients submitted to major vascular procedures $(n=23)$ prohibited us from performing a multivariable analysis of the different potential prognostic factors and kept us from drawing definitive conclusions. However nowadays, there is a paucity of information regarding "vascular" approach in the gynecological setting and such women could be considered not suitable for radical surgery because of vascular involvement thus typically submitted to palliative chemotherapy, affecting their prognosis.

In this paper, once again, a critical prognostic factor seems to be the ability to remove all the tumor with $\mathrm{R} 0$ resection and involvement of the vascular department and/or the iliopsoas muscle may not be an impediment towards attaining this goal (4). Thus, by deliberately sacrificing retroperitoneal vascular structures, sharp dissection of the vessels could become redundant, and the margins, as well as resectability, could be improved to $100 \%$.

In this study, we identified for the first time certain vascular procedures in the surgical treatment of gynecologic cancers and a pre-operative CT-scan score that can accurately predict the need for these procedures allowing for proper planning of surgical treatment. Certainly, the clinical implications of our paper is represented by the importance of choosing aggressive surgery through a multidisciplinary approach (gynecologic oncology surgeon, vascular surgeon, orthopedic surgeon and others) as the best therapeutic option for a subset of patients who otherwise would not be treated. Finally, in our opinion treatment decisions for patients with similar clinical conditions must be made on an individual basis in tertiary referral center and vascular surgery should not be considered as an impairment to gynecological tumor resectability.

\section{Conflicts of Interest}

The Authors have no conflicts of interest to declare.

\section{References}

1 Kehoe S: Treatments for gynecological cancers. Best Pract Res Clin Obstet Gynaecol 20: 985-1000, 2006.

2 Hoeckel M: Long-term experience with (laterally) extended endopelvic resection (LEER) in relapsed pelvic malignancies. Curr Oncol Rep 17(3): 435, 2015.

3 Vizzielli G, Fanfani F, Costantini B, Gallotta V, Scambia G and Fagotti A: External hemipelvectomy as treatment for solitary coxofemoral metastasis from endometrial carcinoma: case report and review of the literature. J Obstet Gynaecol Res 38(5): 892898, 2012.

4 Vizzielli G, Chiantera V, Tinelli G, Fagotti A, Gallotta V, Di Giorgio A, Gueli Alletti S and Scambia G: Out-of-the-box pelvic surgery including iliopsoas resection for recurrent gynecological malignancies: Does that make sense? A single-institution caseseries. Eur J Surg Oncol 43(4): 710-716, 2017.

5 Costantini B, Vizzielli G, Fanfani F, D’Addessi A, Ercoli A, Avenia N, Margariti PA, Gallotta V, Scambia G and Fagotti A: Urologic surgery in gynecologic oncology: a large singleinstitution experience. Eur J Surg Oncol 4O(6): 756-761, 2014.

6 Sinno AK, Li X, Thompson RE, Tanner EJ 3rd, Levinson KL, Stone RL, Temkin SM, Fader AN, Chi DS and Long Roche K: Trends and factors associated with radical cytoreductive surgery in the United States: A case for centralized care. Gynecol Oncol 145(3): 493-499, 2017.

7 Heitz F, Harter P, Alesina PF, Walz MK, Lorenz D, Groeben H, Heikaus S, Fisseler-Eckhoff A, Schneider S, Ataseven B, Kurzeder C, Prader S, Beutel B, Traut A and du Bois A: Pattern of and reason for postoperative residual disease in patients with advanced ovarian cancer following upfront radical debulking surgery. Gynecol Oncol 141(2): 264-270, 2016. 
8 Gallotta V, Fanfani F, Vizzielli G, Panico G, Rossitto C, Gagliardi ML, Margariti PA, Salerno MG, Zannoni GF, Pacelli F, Scambia G and Fagotti A: Douglas peritonectomy compared to recto-sigmoid resection in optimally cytoreduced advanced ovarian cancer patients: analysis of morbidity and oncological outcome. Eur J Surg Oncol 37(12): 1085-1092, 2011.

9 Schwarzbach MH, Hormann Y, Hinz U, Leowardi C, Böckler D, Mechtersheimer G, Friess H, Büchler MW and Allenberg JR: Clinical results of surgery for retroperitoneal sarcoma with major blood vessel involvement. J Vasc Surg 44(1): 46-55, 2006.

10 McKay A, Motamedi M, Temple W, Mack L and Moore R: Vascular reconstruction with the superficial femoral vein following major oncologic resection. J Surg Oncol 96(2): 151159,2007

11 Song TK, Harris EJ Jr., Raghavan S and Norton JA: Major blood vessel reconstruction during sarcoma surgery. Arch Surg 144(9): 817-822, 2009.

12 Oken M, Creech R, Tormey D, Horton J, Davis TE, McFadden ET and Carbone PP: Toxicity and response criteria of the Eastern Cooperative Oncology Group. Am J Clin Oncol 5(6): 649-655, 1982.

13 Strong VE, Selby LV, Sovel M, Disa JJ, Hoskins W, Dematteo R, Scardino P and Jaques DP: Development and assessment of Memorial Sloan Kettering Cancer Center's Surgical Secondary Events grading system. Ann Surg Oncol 22(4): 1061-1067, 2015.
14 Fagotti A, Costantini B, Fanfani F, Vizzielli G, Rossitto C, Lecca A and Scambia G: Risk of postoperative pelvic abscess in major gynecologic oncology surgery: one-year single-institution experience. Ann Surg Oncol 17(9): 2452-2458, 2010.

15 Fagotti A, Ferrandina G, Vizzielli G, Fanfani F, Gallotta V, Chiantera V, Costantini B, Margariti PA, Gueli Alletti S, Cosentino F, Tortorella L and Scambia G: Phase III randomised clinical trial comparing primary surgery versus neoadjuvant chemotherapy in advanced epithelial ovarian cancer with high tumor load (SCORPION trial): Final analysis of peri-operative outcome. Eur J Cancer 59: 22-33, 2016.

16 Grimm C, Polterauer S, Helmy S, Cibula D, Zikan M, Reinthaller A and Tempfer C: A collagen-fibrin patch (Tachosil ${ }^{\circledR}$ ) for the prevention of symptomatic lymphoceles after pelvic lymphadenectomy in women with gynecologic malignancies: a randomized clinical trial. BMC Cancer 14: 635, 2014.

Received September 2, 2017 Revised September 27, 2017 Accepted September 29, 2017 